\title{
II BRAZILIAN CONSENSUS ON GASTRIC CANCER BY THE BRAZILIAN GASTRIC CANCER ASSOCIATION
}

\author{
Il Consenso Brasileiro de Câncer Gástrico realizado pela Associação Brasileira de Câncer Gástrico \\ Leandro Cardoso BARCHI ${ }^{1,2}$, Marcus Fernando Kodama Pertille RAMOS ${ }^{1 \oplus}$, André Roncon DIAS ${ }^{1 \odot}$, \\ Nelson Adami ANDREOLLO ${ }^{3}{ }^{\oplus}$, Antônio Carlos WESTON ${ }^{4 \oplus}$, Laércio Gomes LOURENÇO ${ }^{5 \odot}$, Carlos Alberto \\ MALHEIROS $^{6 \oplus}$, Paulo KASSAB ${ }^{6 \oplus}$, Bruno ZILBERSTEIN ${ }^{1,2 \odot}$ and Consensus members*
}

ABSTRACT - Background: Since the publication of the first Brazilian Consensus on Gastric Cancer (GC) in 2012 carried out by the Brazilian Gastric Cancer Association, new concepts on diagnosis, staging, treatment and follow-up have been incorporated. Aim: This new consensus is to promote an update to professionals working in the fight against GC and to provide guidelines for the management of patients with this condition. Methods: Fiftynine experts answered 67 statements regarding the diagnosis, staging, treatment and prognosis of $\mathrm{GC}$ with five possible alternatives: 1 ) fully agree; 2) partially agree; 3) undecided; 4) disagree and 5) strongly disagree $A$ consensus was adopted when at least $80 \%$ of the sum of the answers "fully agree" and "partially agree" was reached. This article presents only the responses of the participating experts. Comments on each statement, as well as a literature review, will be presented in future publications. Results: Of the 67 statements, there was consensus in 50 (74\%). In 10 declarations, there was 100\% agreement. Conclusion: The gastric cancer treatment has evolved considerably in recent years. This consensus gathers consolidated principles in the last decades, new knowledge acquired recently, as well as promising perspectives on the management of this disease.

HEADINGS: Gastric neoplasms. Gastric cancer. Gastrectomy. Lymphadenectomy. Consensus. Adenocarcinoma.

RESUMO - Racional: Desde a publicação do primeiro Consenso Brasileiro sobre Câncer Gástrico em 2012 realizado pela Associação Brasileira de Câncer Gástrico (ABCG), novos conceitos sobre o diagnóstico, estadiamento, tratamento e seguimento foram incorporados. Objetivo: Promover uma atualização aos profissionais que atuam no combate ao câncer gástrico (CG) e fornecer diretrizes quanto ao manejo dos pacientes portadores desta afecção. Métodos: Cinquenta e nove especialistas responderam 67 declarações sobre o diagnóstico, estadiamento, tratamento e prognóstico do CG com cinco alternativas possíveis: 1) concordo plenamente; 2) concordo parcialmente; 3) indeciso; 4) discordo e 5) discordo fortemente. Foi considerado consenso a concordância de pelo menos $80 \%$ da soma das respostas "concordo plenamente" e "concordo parcialmente". Este artigo apresenta apenas as respostas dos especialistas participantes. Os comentários sobre cada declaração, assim como uma revisão da literatura serão apresentados em publicações futuras. Resultados: Das 67 declarações, houve consenso em 50 (74\%). Em 10 declarações, houve concordância de 100\%. Conclusão: O tratamento do câncer gástrico evoluiu consideravelmente nos últimos anos. Este consenso reúne princípios consolidados nas últimas décadas, novos conhecimentos adquiridos recentemente, assim como perspectivas promissoras sobre o manejo desta doença.

DESCRITORES - Neoplasias gástricas. Câncer gástrico. Gastrectomia. Linfadenectomia. Consenso. Adenocarcinoma.

Central message
The II Brazilian Consensus on Gastric Cancer gathers
consolidated concepts with new information and
knowledge acquired recently, allowing better
outcomes in the treatment of this disease.

\section{Perspective}

The II Brazilian Consensus on Gastric Cancer gathers the opinion of renowned Brazilian experts who work in the fight against gastric cancer. Among them are surgeons, oncologists, endoscopists and pathologists. The answers to the statements contained in this consensus, allow an update on the management of care for patients with gastric cancer.

From the ${ }^{1}$ Hospital das Clínicas, Faculdade de Medicina, Universidade de São Paulo, São Paulo, SP, Brasil; ${ }^{2}$ Faculdade de Medicina São Leopoldo Mandic, Campinas, SP, Brasil; ${ }^{3}$ Universidade Estadual de Campinas, Campinas, SP, Brasil; ${ }^{4}$ Departamento de Cirurgia, Santa Casa de Porto Alegre, Porto Alegre, RS, Brasil; ${ }^{5}$ Universidade Federal de São Paulo, São Paulo, SP, Brasil; ${ }^{6}$ Departamento de Cirurgia, Santa Casa de São Paulo, São Paulo, SP, Brasil ('Hospital das Clínicas, Faculty of Medicine, University of São Paulo, São Paulo, SP, Brazil; ${ }^{2}$ Faculty of Medicine São Leopoldo Mandic, Campinas, SP, Brazil; ${ }^{3}$ State University of Campinas, Campinas, SP, Brazil; ${ }^{4}$ Department of Surgery, Santa Casa de Porto Alegre, Porto Alegre, RS, Brazil; ${ }^{5}$ Federal University of São Paulo, São Paulo, SP, Brazil; ' 6 Department of Surgery, Santa Casa de São Paulo, São Paulo, SP, Brazil).

How to cite this article: Barchi LC, Ramos MFKP, Dias AR, Andreollo NA, Weston AC, Lourenço LG, Malheiros CA, Kassab P, Zilberstein B. II Brazilian Consensus on Gastric Cancer by the Brazilian Gastric Cancer Association. ABCD Arq Bras Cir Dig. 2020;33(2):e1514. DOI: /10.1590/0102-672020190001e1514

Correspondence:

Leandro Cardoso Barchi

E-mail: leandrobarchi@hotmail.com

Financial source: none

Conflict of interest: none

Received for publication: 31/03/2020

Accepted for publication: 02/06/2020 
INTRODUCTION

The incidence of gastric cancer (GC) has decreased around the world. Once the second most common type of cancer, currently CG is the fifth, after lung, breast, prostate and colorectal ${ }^{6,10,15}$. Its incidence has dropped in the last 50 years due to the improvement in basic sanitation conditions, the use of refrigerators and consumption improvement of fresh fruits and vegetables and less salt intake, which was then widely used to preserve food. Other factors that contributed to this decline were the eradication of Helicobacter pylori and the screening intensification in several countries ${ }^{14}$.

Despite that, the mortality rate remains high. In 2018, CG recovered the second place in cancer deaths around the world, surpassing liver cancer again and placing only after lung cancer ${ }^{6}$. In Brazil, it is the third most common type among men and the fifth among women ${ }^{1}$. According to the Instituto Nacional do Câncer (INCA) data, it is estimated that in the $2018 / 2019$ biennium 21,290 new cases were diagnosed (13,540 in men and 7,750 in women) and about 15,000 deaths in 2017 related to it ${ }^{11}$.

Adenocarcinoma is the most common histological type, accountable for about $90-95 \%$ of cases. Other less common malignant neoplasms of the stomach such as lymphoma (4\%), neuroendocrine tumor (3\%) and gastrointestinal stromal tumor (GIST) will not be addressed in this consensus ${ }^{5,9}$.

Due to the continental dimension of Brazil, the incidence, management and prognosis varies widely according to the different regions of the country. In 2013, the first Brazilian Consensus on Gastric Cancer was published by the Brazilian Gastric Cancer Association (BGCA). This consensus aimed to unify and standardize the diagnosis and management of this condition ${ }^{21}$. Undoubtedly, the publication of this guideline made it possible to spread knowledge among medical professionals and, consequently, improve care and increase survival of these patients.

However, since the publication of the first Consensus, many aspects related to $C G$ have changed ${ }^{16,17}$. It lists: new TNM staging classification was implemented by the American Joint Committee on Cancerand Union for International Cancer Control (AJCC/UICC) ${ }^{18}$; the agreement between the West and the East on the pivotal role of D2 lymphadenectomy as a standard surgical treatment; the increasing role of multimodal therapy (neoadjuvant, perioperative and adjuvant chemotherapy/radiotherapy); the replacement of the systematic removal of the lymph node stations by the minimum number of 15 lymph nodes in the D2 lymphadenectomy; the indication for multivisceral resection (splenectomy); the endoscopic treatment in early GC and the role of minimally invasive surgery (laparoscopic or robotic) as an alternative surgical approach.

Thus, in view of the evidence gathered in recent years, it is opportune to update the Brazilian guidelines on CG through a new consensus. It is important to note that the guidelines hereby presented are not arbitrary and, therefore, it is up to each medical assistant/multidisciplinaryteam to adopt the best conduct according to local reality and the resource availability, as long as they are indeed beneficial to the patient.

\section{METHODS}

The Brazilian Gastric Cancer Association celebrated 20 years of its foundation in 1999. To solemnize this date, a commemorative day was held in Porto Alegre (RS) on August 16, when there was the opportunity to debate and present the results of this new Consensus. Three months before this event, a group of ABCG experts created $67 \mathrm{GC}$ statements on diagnosis, staging, treatment and prognosis with five possible alternatives: 1) fully agree; 2) partially agree; 3) undecided; 4) disagree and 5) strongly disagree. These statements were sent to 59 specialists in GC treatment from all regions of Brazil (surgeons, oncologists, endoscopists, pathologists, etc.), embracing more than 20 universities institutions. The participants were able to mark only one answer. A consensus was achieved when the sum of "fully agree" and "partially agree" reached at least $80 \%$. This article presents only the responses of the participating experts. Comments on each statement, as well as a literature review, will be presented in future publications.

\section{RESULTS}

Of the 67 statements presented, a consensus was reached in 50 (74\%). In 10 statements, there was 100\% agreement.

\section{Diagnosis statements}

\section{Statement 1}

The main method of gastric cancer diagnosis is through an upper gastrointestinal endoscopy with biopsy. The endoscopic examination report must contain precise information regarding location (s) of the lesion (s), approximate size, extent, infiltration, distance from the esophageal-gastric transition and the pylorus, detailing the places where the biopsies were performed.

\begin{tabular}{|c|c|c|c|c|c|c|}
\hline Fully agree & $\begin{array}{c}\text { Partially } \\
\text { agree }\end{array}$ & Undecided & Disagree & $\begin{array}{l}\text { Strongly } \\
\text { disagree }\end{array}$ & Total & Consensus \\
\hline $98 \%$ & $2 \%$ & $0 \%$ & $0 \%$ & $0 \%$ & $100 \%$ & $100 \%$ \\
\hline
\end{tabular}

\section{Statement 2}

In case of high suspicion of gastric cancer and repeatedly negative biopsies collected by upper gastrointestinal endoscopy (including macrobiopsies), the diagnosis can be made through endoscopic resection or surgery.

\begin{tabular}{|c|c|c|c|c|c|c|}
\hline Fully agree & $\begin{array}{l}\text { Partially } \\
\text { agree }\end{array}$ & Undecided & Disagree & $\begin{array}{l}\text { Strongly } \\
\text { disagree }\end{array}$ & Total & Consensus \\
\hline $58 \%$ & $36 \%$ & $0 \%$ & $2 \%$ & $4 \%$ & $100 \%$ & $94 \%$ \\
\hline
\end{tabular}

\section{Statement 3}

Ultrasound upper endoscopy is not indicated when there are clear endoscopic signs that the cancer is invasive. It should be used when there is any doubt about the early aspect of GC. It allows to evaluate the degree of tumor invasion in the gastric wall and the presence of suspicious lymph nodes for metastases.

\begin{tabular}{|c|c|c|c|c|c|c|}
\hline Fully agree & $\begin{array}{c}\text { Partially } \\
\text { agree }\end{array}$ & Undecided & Disagree & $\begin{array}{l}\text { Strongly } \\
\text { disagree }\end{array}$ & Total & Consensus \\
\hline $72 \%$ & $24 \%$ & $2 \%$ & $2 \%$ & $0 \%$ & $100 \%$ & $96 \%$ \\
\hline
\end{tabular}

\section{Statement 4}

The main staging method is computed tomography of the chest, abdomen and pelvis.

\begin{tabular}{|c|c|c|c|c|c|c|}
\hline Fully agree & $\begin{array}{l}\text { Partially } \\
\text { agree }\end{array}$ & Undecided & Disagree & $\begin{array}{l}\text { Strongly } \\
\text { disagree }\end{array}$ & Total & Consensus \\
\hline $90 \%$ & $10 \%$ & $0 \%$ & $0 \%$ & $0 \%$ & $100 \%$ & $100 \%$ \\
\hline
\end{tabular}

Statement 5

Positron emission tomography (PET-CT) and nuclear magnetic resonance (MRI) should be used only in selected cases.

\begin{tabular}{|c|c|c|c|c|c|c|}
\hline Fully agree & $\begin{array}{l}\text { Partially } \\
\text { agree }\end{array}$ & Undecided & Disagree & $\begin{array}{l}\text { Strongly } \\
\text { disagree }\end{array}$ & Total & Consensus \\
\hline $92 \%$ & $8 \%$ & $0 \%$ & $0 \%$ & $0 \%$ & $100 \%$ & $100 \%$ \\
\hline
\end{tabular}

\section{Statement 6}

PET-CT can be used in well-differentiated tumors or in the proximal third.

\begin{tabular}{|c|c|c|c|c|c|c|}
\hline Fully agree & $\begin{array}{l}\text { Partially } \\
\text { agree }\end{array}$ & Undecided & Disagree & $\begin{array}{l}\text { Strongly } \\
\text { disagree }\end{array}$ & Total & Consensus \\
\hline $44 \%$ & $30 \%$ & $6 \%$ & $16 \%$ & $4 \%$ & $100 \%$ & $74 \%$ \\
\hline
\end{tabular}


Statement 7

Staging laparoscopy should be performed in cases where there is uncertainty in computed tomography regarding the presence of peritoneal carcinomatosis or when multidisciplinary treatment is planned.

\begin{tabular}{|c|c|c|c|c|c|c|}
\hline Fully agree & $\begin{array}{c}\text { Partially } \\
\text { agree }\end{array}$ & Undecided & Disagree & $\begin{array}{l}\text { Strongly } \\
\text { disagree }\end{array}$ & Total & Consensus \\
\hline $80 \%$ & $18 \%$ & $0 \%$ & $2 \%$ & $0 \%$ & $100 \%$ & $98 \%$ \\
\hline
\end{tabular}

\section{Statement 8}

Peritoneal washing with oncotic cytology should be performed in all cases during staging laparoscopy and/or surgery. It may be omitted if there is frank peritoneal carcinomatosis.

\begin{tabular}{|c|c|c|c|c|c|c|}
\hline Fully agree & $\begin{array}{c}\text { Partially } \\
\text { agree }\end{array}$ & Undecided & Disagree & $\begin{array}{l}\text { Strongly } \\
\text { disagree }\end{array}$ & Total & Consensus \\
\hline & $24 \%$ & $4 \%$ & $0 \%$ & $0 \%$ & $100 \%$ & $96 \%$ \\
\hline
\end{tabular}

\section{Statement 9}

Analysis of serum tumor markers (CA19.9, CEA, CA 72.4) should be performed in all cases of gastric cancer.

\begin{tabular}{|c|c|c|c|c|c|c|}
\hline Fully agree & $\begin{array}{l}\text { Partially } \\
\text { agree }\end{array}$ & Undecided & Disagree & $\begin{array}{l}\text { Strongly } \\
\text { disagree }\end{array}$ & Total & Consensus \\
\hline $18 \%$ & $38 \%$ & $6 \%$ & $26 \%$ & $12 \%$ & $100 \%$ & $56 \%$ \\
\hline
\end{tabular}

\section{Declaration 10}

Currently, the staging that must be adopted is the UICC/ AJCC TNM $8^{\text {th }}$ edition.

\begin{tabular}{|c|c|c|c|c|c|c|}
\hline Fully agree & $\begin{array}{c}\text { Partially } \\
\text { agree }\end{array}$ & Undecided & Disagree & $\begin{array}{l}\text { Strongly } \\
\text { disagree }\end{array}$ & Total & Consensus \\
\hline & $4 \%$ & $0 \%$ & $0 \%$ & $0 \%$ & $100 \%$ & $100 \%$ \\
\hline
\end{tabular}

\section{Treatment statements}

Statement 11

Multidisciplinary therapeutic planning (surgeon, endoscopist, general clinician, oncologist, radiologist and pathologist) is recommended before starting any type of treatment.

\begin{tabular}{|c|c|c|c|c|c|c|}
\hline Fully agree & $\begin{array}{c}\text { Partially } \\
\text { agree }\end{array}$ & Undecided & Disagree & $\begin{array}{l}\text { Strongly } \\
\text { disagree }\end{array}$ & Total & Consensus \\
\hline $70 \%$ & $20 \%$ & $0 \%$ & $10 \%$ & $0 \%$ & $100 \%$ & $90 \%$ \\
\hline
\end{tabular}

\section{Statement 12}

Patients who had weight lost greater than $10 \%$ of their usual weight in the past six months should receive some form of nutritional therapy before starting any treatment.

\begin{tabular}{|c|c|c|c|c|c|c|}
\hline Fully agree & $\begin{array}{c}\text { Partially } \\
\text { agree }\end{array}$ & Undecided & Disagree & $\begin{array}{l}\text { Strongly } \\
\text { disagree }\end{array}$ & Total & \\
\hline $68 \%$ & $32 \%$ & $0 \%$ & $0 \%$ & $0 \%$ & $100 \%$ & $100 \%$ \\
\hline
\end{tabular}

\section{Statement 13}

Endoscopic resection is indicated in well-differentiated adenocarcinoma tumors, restricted to the mucosa (T1a), less than $2 \mathrm{~cm}$ in its longest axis and not ulcerated.

\begin{tabular}{|c|c|c|c|c|c|c|}
\hline Fully agree & $\begin{array}{c}\text { Partially } \\
\text { agree }\end{array}$ & Undecided & Disagree & $\begin{array}{l}\text { Strongly } \\
\text { disagree }\end{array}$ & Total & Consensus \\
\hline $94 \%$ & $6 \%$ & $0 \%$ & $0 \%$ & $0 \%$ & $100 \%$ & $100 \%$ \\
\hline
\end{tabular}

\section{Statement 14}

Early lesions with invasion of the submucosal layer, ulcerated, diffuse type and larger than $2 \mathrm{~cm}$ are exception criteria for endoscopic resection and should be adopted only in patients at high surgical risk.

\begin{tabular}{|c|c|c|c|c|c|c|}
\hline Fully agree & $\begin{array}{l}\text { Partially } \\
\text { agree }\end{array}$ & Undecided & Disagree & $\begin{array}{l}\text { Strongly } \\
\text { disagree }\end{array}$ & Total & Consensus \\
\hline & $16 \%$ & $2 \%$ & $6 \%$ & $0 \%$ & $100 \%$ & $92 \%$ \\
\hline
\end{tabular}

Statement 15

Endoscopic submucosal dissection (ESD) is recommended as the treatment of choice for most superficial gastric tumors.

\begin{tabular}{|c|c|c|c|c|c|c|}
\hline Fully agree & $\begin{array}{l}\text { Partially } \\
\text { agree }\end{array}$ & Undecided & Disagree & $\begin{array}{l}\text { Strongly } \\
\text { disagree }\end{array}$ & Total & Consensus \\
\hline $42 \%$ & $34 \%$ & $8 \%$ & $8 \%$ & $8 \%$ & $100 \%$ & $76 \%$ \\
\hline
\end{tabular}

\section{Statement 16}

For tumors that do not meet endoscopic resection indication (T1b), surgery is indicated. In these cases, the recommended lymph node dissection is the removal of the perigastric lymph nodes (D1) in well-differentiated tumors smaller than $1.5 \mathrm{~cm}$ and associated with the removal of some lymph nodes in the N2 chain (D1 +) for undifferentiated tumors smaller than $1.5 \mathrm{~cm}$.

\begin{tabular}{|c|c|c|c|c|c|c|}
\hline Fully agree & $\begin{array}{c}\text { Partially } \\
\text { agree }\end{array}$ & Undecided & Disagree & $\begin{array}{l}\text { Strongly } \\
\text { disagree }\end{array}$ & Total & Consensus \\
\hline $42 \%$ & $34 \%$ & $2 \%$ & $18 \%$ & $4 \%$ & $100 \%$ & $76 \%$ \\
\hline
\end{tabular}

Statement 17 is indicated.

In stage IB-III tumors (T2-4 any N), D2 lymph node dissection

\begin{tabular}{|c|c|c|c|c|c|c|}
\hline Fully agree & $\begin{array}{l}\text { Partially } \\
\text { agree }\end{array}$ & Undecided & Disagree & $\begin{array}{l}\text { Strongly } \\
\text { disagree }\end{array}$ & Total & Consensus \\
\hline $90 \%$ & $8 \%$ & $2 \%$ & $0 \%$ & $0 \%$ & $100 \%$ & $98 \%$ \\
\hline
\end{tabular}

Statement 18

The UICC / AJCC recommends a minimum of 15 harvested lymph nodes to allow correct staging.

\begin{tabular}{|c|c|c|c|c|c|c|}
\hline Fully agree & $\begin{array}{l}\text { Partially } \\
\text { agree }\end{array}$ & Undecided & Disagree & $\begin{array}{l}\text { Strongly } \\
\text { disagree }\end{array}$ & Total & Consensus \\
\hline $78 \%$ & $14 \%$ & $0 \%$ & $6 \%$ & $2 \%$ & $100 \%$ & $92 \%$ \\
\hline
\end{tabular}

\section{Statement 19}

D2 lymphadenectomy recommends at least 25 harvested lymph nodes.

\begin{tabular}{|c|c|c|c|c|c|c|}
\hline Fully agree & $\begin{array}{c}\text { Partially } \\
\text { agree }\end{array}$ & Undecided & Disagree & $\begin{array}{l}\text { Strongly } \\
\text { disagree }\end{array}$ & Total & Consensus \\
\hline $54 \%$ & $22 \%$ & $4 \%$ & $18 \%$ & $2 \%$ & $100 \%$ & $76 \%$ \\
\hline
\end{tabular}

\section{Statament 20}

It is recommended at the end of each operation that a member of the surgical team send the surgical specimen for the pathological analysis with all the separate and identified lymph node chains.

\begin{tabular}{|c|c|c|c|c|c|c|}
\hline Fully agree & $\begin{array}{l}\text { Partially } \\
\text { agree }\end{array}$ & Undecided & Disagree & $\begin{array}{l}\text { Strongly } \\
\text { disagree }\end{array}$ & Total & Consensus \\
\hline $72 \%$ & $18 \%$ & $2 \%$ & $8 \%$ & $0 \%$ & $100 \%$ & $90 \%$ \\
\hline
\end{tabular}

Statement 21

Lymphadenectomies more extended than D2 (D2 + or D3) should be reserved only in selected cases.

\begin{tabular}{|c|c|c|c|c|c|c|}
\hline Fully agree & $\begin{array}{c}\text { Partially } \\
\text { agree }\end{array}$ & Undecided & Disagree & $\begin{array}{l}\text { Strongly } \\
\text { disagree }\end{array}$ & Total & Consensus \\
\hline $84 \%$ & $8 \%$ & $2 \%$ & $6 \%$ & $0 \%$ & $100 \%$ & $92 \%$ \\
\hline
\end{tabular}

Statement 22

Subtotal gastrectomy should be performed on distal tumors or in cases where the proximal margin is at least $5 \mathrm{~cm}$ between the tumor and the esophagogastric transition.

\begin{tabular}{|c|c|c|c|c|c|c|}
\hline Fully agree & $\begin{array}{l}\text { Partially } \\
\text { agree }\end{array}$ & Undecided & Disagree & $\begin{array}{l}\text { Strongly } \\
\text { disagree }\end{array}$ & Total & Consensus \\
\hline $70 \%$ & $26 \%$ & $2 \%$ & $2 \%$ & $0 \%$ & $100 \%$ & $96 \%$ \\
\hline
\end{tabular}


Statement 23

In diffuse tumors, a proximal margin of at least $8 \mathrm{~cm}$ is recommended.

\begin{tabular}{|c|c|c|c|c|c|c|}
\hline Fully agree & $\begin{array}{l}\text { Partially } \\
\text { agree }\end{array}$ & Undecided & Disagree & $\begin{array}{l}\text { Strongly } \\
\text { disagree }\end{array}$ & Total & Consensus \\
\hline $34 \%$ & $38 \%$ & $8 \%$ & $16 \%$ & $4 \%$ & $100 \%$ & $72 \%$ \\
\hline
\end{tabular}

\section{Statement 24}

In tumors invading the distal esophagus, the resection margin must be confirmed by frozen biopsy.

\begin{tabular}{|c|c|c|c|c|c|c|}
\hline Fully agree & $\begin{array}{c}\text { Partially } \\
\text { agree }\end{array}$ & Undecided & Disagree & $\begin{array}{l}\text { Strongly } \\
\text { disagree }\end{array}$ & Total & Consensus \\
\hline $72 \%$ & $22 \%$ & $2 \%$ & $2 \%$ & $2 \%$ & $100 \%$ & $94 \%$ \\
\hline
\end{tabular}

Statement 25

Total gastrectomy is recommended for proximal tumors and early multicentric tumors.

\begin{tabular}{|c|c|c|c|c|c|c|}
\hline Fully agree & $\begin{array}{l}\text { Partially } \\
\text { agree }\end{array}$ & Undecided & Disagree & $\begin{array}{l}\text { Strongly } \\
\text { disagree }\end{array}$ & Total & Consensus \\
\hline $74 \%$ & $26 \%$ & $0 \%$ & $0 \%$ & $0 \%$ & $100 \%$ & $100 \%$ \\
\hline
\end{tabular}

\section{Statement 26}

Splenectomy should be performed only in advanced tumors from the greater curvature of the proximal stomach, when there is invasion of the spleen or if there is evident lymph node involvement of the splenic hilum.

\begin{tabular}{|c|c|c|c|c|c|c|}
\hline Fully agree & $\begin{array}{l}\text { Partially } \\
\text { agree }\end{array}$ & Undecided & Disagree & $\begin{array}{l}\text { Strongly } \\
\text { disagree }\end{array}$ & Total & Consensus \\
\hline $76 \%$ & $20 \%$ & $4 \%$ & $0 \%$ & $0 \%$ & $100 \%$ & $96 \%$ \\
\hline
\end{tabular}

\section{Statement 27}

Patients with unresectable or marginally resectable lesions may be candidates for conversion therapy, which consists of chemotherapy followed by surgery to achieve R0 resection.

\begin{tabular}{|c|c|c|c|c|c|c|}
\hline Fully agree & $\begin{array}{l}\text { Partially } \\
\text { agree }\end{array}$ & Undecided & Disagree & $\begin{array}{l}\text { Strongly } \\
\text { disagree }\end{array}$ & Total & Consensus \\
\hline $78 \%$ & $20 \%$ & $0 \%$ & $2 \%$ & $0 \%$ & $100 \%$ & $98 \%$ \\
\hline
\end{tabular}

\section{Statement 28}

Duodenopancreatectomy can be indicated in cases of locally advanced gastric cancer, T4N0-2M0, young patients and with low surgical risk.

\begin{tabular}{|c|c|c|c|c|c|c|}
\hline Fully agree & $\begin{array}{l}\text { Partially } \\
\text { agree }\end{array}$ & Undecided & Disagree & $\begin{array}{l}\text { Strongly } \\
\text { disagree }\end{array}$ & Total & Consensus \\
\hline $58 \%$ & $32 \%$ & $4 \%$ & $6 \%$ & $0 \%$ & $100 \%$ & $90 \%$ \\
\hline
\end{tabular}

\section{Statement 29}

Hepatectomy is indicated in infiltrative liver tumors (T4b) without peritoneal carcinomatosis.

\begin{tabular}{|c|c|c|c|c|c|c|}
\hline Fully agree & $\begin{array}{c}\text { Partially } \\
\text { agree }\end{array}$ & Undecided & Disagree & $\begin{array}{l}\text { Strongly } \\
\text { disagree }\end{array}$ & Total & Consensus \\
\hline $68 \%$ & $30 \%$ & $0 \%$ & $2 \%$ & $0 \%$ & $100 \%$ & $98 \%$ \\
\hline
\end{tabular}

\section{Statement 30}

Patients with single liver metastasis may be eligible for surgery after a multidisciplinary evaluation.

\begin{tabular}{|c|c|c|c|c|c|c|}
\hline Fully agree & $\begin{array}{c}\text { Partially } \\
\text { agree }\end{array}$ & Undecided & Disagree & $\begin{array}{l}\text { Strongly } \\
\text { disagree }\end{array}$ & Total & Consensus \\
\hline $62 \%$ & $24 \%$ & $8 \%$ & $6 \%$ & $0 \%$ & $100 \%$ & $86 \%$ \\
\hline
\end{tabular}

Statement 31

The combined resection of adjacent or multivisceral organs can be performed, as long as the patient is in good clinical condition and, preferably, R0 resection is achieved.

\begin{tabular}{|c|c|c|c|c|c|c|}
\hline Fully agree & $\begin{array}{l}\text { Partially } \\
\text { agree }\end{array}$ & Undecided & Disagree & $\begin{array}{l}\text { Strongly } \\
\text { disagree }\end{array}$ & Total & Consensus \\
\hline $88 \%$ & $8 \%$ & $0 \%$ & $4 \%$ & $0 \%$ & $100 \%$ & $96 \%$ \\
\hline
\end{tabular}

\section{Statement 32}

In patients considered $\mathrm{M} 1$, palliative gastric resection may occasionally be performed in cases of obstruction, bleeding or perforation.

\begin{tabular}{|c|c|c|c|c|c|c|}
\hline Fully agree & $\begin{array}{l}\text { Partially } \\
\text { agree }\end{array}$ & Undecided & Disagree & $\begin{array}{l}\text { Strongly } \\
\text { disagree }\end{array}$ & Total & Consensus \\
\hline $90 \%$ & $10 \%$ & $0 \%$ & $0 \%$ & $0 \%$ & $100 \%$ & $100 \%$ \\
\hline
\end{tabular}

\section{Statement 33}

Palliative gastric resection in asymptomatic patients is not indicated as the first treatment approach.

\begin{tabular}{|c|c|c|c|c|c|c|}
\hline Fully agree & $\begin{array}{c}\text { Partially } \\
\text { agree }\end{array}$ & Undecided & Disagree & $\begin{array}{l}\text { Strongly } \\
\text { disagree }\end{array}$ & Total & Consensus \\
\hline $70 \%$ & $16 \%$ & $4 \%$ & $10 \%$ & $0 \%$ & $100 \%$ & $86 \%$ \\
\hline
\end{tabular}

\section{Statement 34}

Partial omentectomy (up to $3-5 \mathrm{~cm}$ from the gastroepiploic arcade) can be performed on $\mathrm{T} 1 / \mathrm{T} 2$ tumors and total omentectomy must be performed on T3/T4 tumors.

\begin{tabular}{|c|c|c|c|c|c|c|}
\hline Fully agree & $\begin{array}{c}\text { Partially } \\
\text { agree }\end{array}$ & Undecided & Disagree & $\begin{array}{l}\text { Strongly } \\
\text { disagree }\end{array}$ & Total & Consensus \\
\hline $48 \%$ & $30 \%$ & $14 \%$ & $6 \%$ & $2 \%$ & $100 \%$ & $78 \%$ \\
\hline
\end{tabular}

\section{Statement 35}

Bursectomy should be performed only on T4 tumors arising from the posterior gastric wall.

\begin{tabular}{|c|c|c|c|c|c|c|}
\hline Fully agree & $\begin{array}{c}\text { Partially } \\
\text { agree }\end{array}$ & Undecided & Disagree & $\begin{array}{l}\text { Strongly } \\
\text { disagree }\end{array}$ & Total & Consensus \\
\hline $48 \%$ & $32 \%$ & $8 \%$ & $12 \%$ & $0 \%$ & $100 \%$ & $80 \%$ \\
\hline
\end{tabular}

\section{Statement 36}

Prophylactic total gastrectomy is indicated in confirmed familial hereditary gastric cancer with the CDh1 gene mutation.

\begin{tabular}{|c|c|c|c|c|c|c|}
\hline Fully agree & $\begin{array}{c}\text { Partially } \\
\text { agree }\end{array}$ & Undecided & Disagree & $\begin{array}{l}\text { Strongly } \\
\text { disagree }\end{array}$ & Total & Consensus \\
\hline $72 \%$ & $28 \%$ & $0 \%$ & $0 \%$ & $0 \%$ & $100 \%$ & $100 \%$ \\
\hline
\end{tabular}

\section{Statement 37}

Laparoscopic subtotal gastrectomy can be performed in distal third early GC.

\begin{tabular}{|c|c|c|c|c|c|c|}
\hline Fully agree & $\begin{array}{c}\text { Partially } \\
\text { agree }\end{array}$ & Undecided & Disagree & $\begin{array}{l}\text { Strongly } \\
\text { disagree }\end{array}$ & Total & Consensus \\
\hline $90 \%$ & $8 \%$ & $2 \%$ & $0 \%$ & $0 \%$ & $100 \%$ & $98 \%$ \\
\hline
\end{tabular}

\section{Statement 38}

Laparoscopic subtotal gastrectomy can be performed in distal third advanced gastric cancer.

\begin{tabular}{|c|c|c|c|c|c|c|}
\hline Fully agree & $\begin{array}{l}\text { Partially } \\
\text { aqree }\end{array}$ & Undecided & Disagree & $\begin{array}{l}\text { Strongly } \\
\text { disagree }\end{array}$ & Total & Consensus \\
\hline $52 \%$ & $40 \%$ & $8 \%$ & $0 \%$ & $0 \%$ & $100 \%$ & $92 \%$ \\
\hline
\end{tabular}

Statement 39

Laparoscopic total gastrectomy can be performed in upper third early gastric cancer.

\begin{tabular}{|c|c|c|c|c|c|c|}
\hline Fully agree & $\begin{array}{l}\text { Partially } \\
\text { agree }\end{array}$ & Undecided & Disagree & $\begin{array}{l}\text { Strongly } \\
\text { disagree }\end{array}$ & Total & Consensus \\
\hline $62 \%$ & $28 \%$ & $8 \%$ & $2 \%$ & $0 \%$ & $100 \%$ & $90 \%$ \\
\hline
\end{tabular}


Statement 40

Laparoscopic total gastrectomy can be performed in upper third advanced GC.

\begin{tabular}{|c|c|c|c|c|c|c|}
\hline Fully agree & $\begin{array}{c}\text { Partially } \\
\text { agree }\end{array}$ & Undecided & Disagree & $\begin{array}{l}\text { Strongly } \\
\text { disagree }\end{array}$ & Total & Consensus \\
\hline & $38 \%$ & $16 \%$ & $8 \%$ & $0 \%$ & $100 \%$ & $76 \%$ \\
\hline
\end{tabular}

Statement 41

The use of the robotic platform has the same indications and results as laparoscopy.

\begin{tabular}{|c|c|c|c|c|c|c|}
\hline Fully agree & $\begin{array}{c}\text { Partially } \\
\text { agree }\end{array}$ & Undecided & Disagree & $\begin{array}{l}\text { Strongly } \\
\text { disagree }\end{array}$ & Total & \\
\hline & $26 \%$ & $8 \%$ & $2 \%$ & $0 \%$ & $100 \%$ & $90 \%$ \\
\hline
\end{tabular}

Statement 42

In Siewert type III adenocarcinomas, the standard surgery is total gastrectomy with distal esophagectomy.

\begin{tabular}{|c|c|c|c|c|c|c|}
\hline Fully agree & $\begin{array}{l}\text { Partially } \\
\text { agree }\end{array}$ & Undecided & Disagree & $\begin{array}{l}\text { Strongly } \\
\text { disagree }\end{array}$ & Total & Consensus \\
\hline $82 \%$ & $14 \%$ & $2 \%$ & $2 \%$ & $0 \%$ & $100 \%$ & $96 \%$ \\
\hline
\end{tabular}

\section{Statement 43}

In Siewert type II adenocarcinomas, the standard surgery is transthoracic esophagectomy (thoracoscopy) with proximal gastrectomy and gastric conduit.

\begin{tabular}{|c|c|c|c|c|c|c|}
\hline Fully agree & $\begin{array}{c}\text { Partially } \\
\text { agree }\end{array}$ & Undecided & Disagree & $\begin{array}{l}\text { Strongly } \\
\text { disagree }\end{array}$ & Total & Consensus \\
\hline $32 \%$ & $32 \%$ & $10 \%$ & $22 \%$ & $4 \%$ & $100 \%$ & $64 \%$ \\
\hline
\end{tabular}

\section{Statement 44}

In Siewert type I adenocarcinomas, the standard surgery is transthoracic esophagectomy (thoracoscopy) with proximal gastrectomy and gastric conduit.

\begin{tabular}{|c|c|c|c|c|c|c|}
\hline Fully agree & $\begin{array}{c}\text { Partially } \\
\text { agree }\end{array}$ & Undecided & Disagree & $\begin{array}{l}\text { Strongly } \\
\text { disagree }\end{array}$ & Total & Consensus \\
\hline & $18 \%$ & $4 \%$ & $4 \%$ & $0 \%$ & $100 \%$ & $92 \%$ \\
\hline
\end{tabular}

\section{Statement 45}

Transmediastinal esophagectomy should be reserved for patients with poor or borderline clinical conditions and/or inability to access the thoracic cavity.

\begin{tabular}{|c|c|c|c|c|c|c|}
\hline Fully agree & $\begin{array}{c}\text { Partially } \\
\text { agree }\end{array}$ & Undecided & Disagree & $\begin{array}{c}\text { Strongly } \\
\text { disagree }\end{array}$ & Total & Consensus \\
\hline $36 \%$ & $42 \%$ & $6 \%$ & $14 \%$ & $2 \%$ & $100 \%$ & $78 \%$ \\
\hline
\end{tabular}

\section{Statement 46}

It is recommended that following an esophagectomy, the esophagogastrostomy should be performed preferably in the cervical region.

\begin{tabular}{|c|c|c|c|c|c|c|}
\hline Fully agree & $\begin{array}{l}\text { Partially } \\
\text { agree }\end{array}$ & Undecided & Disagree & $\begin{array}{l}\text { Strongly } \\
\text { disagree }\end{array}$ & Total & \\
\hline $66 \%$ & $24 \%$ & $6 \%$ & $2 \%$ & $2 \%$ & $100 \%$ & $90 \%$ \\
\hline
\end{tabular}

Statement 47

Routine abdominal drain(s) are recommended for all gastric resections.

\begin{tabular}{|c|c|c|c|c|c|c|}
\hline Fully agree & $\begin{array}{c}\text { Partially } \\
\text { agree }\end{array}$ & Undecided & Disagree & $\begin{array}{l}\text { Strongly } \\
\text { disagree }\end{array}$ & Total & Consensus \\
\hline $36 \%$ & $34 \%$ & $6 \%$ & $22 \%$ & $2 \%$ & $100 \%$ & $70 \%$ \\
\hline
\end{tabular}

Statement 48

The duodenal stump should preferably be closed using mechanical suture.

\begin{tabular}{|c|c|c|c|c|c|c|}
\hline Fully agree & $\begin{array}{l}\text { Partially } \\
\text { aqree }\end{array}$ & Undecided & Disagree & $\begin{array}{l}\text { Strongly } \\
\text { disaqree }\end{array}$ & Total & Consensus \\
\hline $58 \%$ & $26 \%$ & $6 \%$ & $8 \%$ & $2 \%$ & $100 \%$ & $84 \%$ \\
\hline
\end{tabular}

Statement 49

There is no clear scientific evidence that reinforcement of the duodenal stump stapling line reduces the incidence of fistulas.

\begin{tabular}{|c|c|c|c|c|c|c|}
\hline Fully agree & $\begin{array}{c}\text { Partially } \\
\text { agree }\end{array}$ & Undecided & Disagree & $\begin{array}{l}\text { Strongly } \\
\text { disagree }\end{array}$ & Total & Consensus \\
\hline $60 \%$ & $24 \%$ & $12 \%$ & $4 \%$ & $0 \%$ & $100 \%$ & $84 \%$ \\
\hline
\end{tabular}

Statement 50

In subtotal and total gastrectomies, digestive transit should preferably be reconstructed by Roux-en-Y derivation.

\begin{tabular}{|c|c|c|c|c|c|c|}
\hline Fully agree & $\begin{array}{c}\text { Partially } \\
\text { agree }\end{array}$ & Undecided & Disagree & $\begin{array}{l}\text { Strongly } \\
\text { disagree }\end{array}$ & Total & Consensus \\
\hline $84 \%$ & $12 \%$ & $4 \%$ & $0 \%$ & $0 \%$ & $100 \%$ & $96 \%$ \\
\hline
\end{tabular}

\section{Statement 51}

Gastrojejunostomy and esophagojejunostomy should preferably be performed with mechanical suture.

\begin{tabular}{|c|c|c|c|c|c|c|}
\hline Fully agree & $\begin{array}{c}\text { Partially } \\
\text { agree }\end{array}$ & Undecided & Disagree & $\begin{array}{l}\text { Strongly } \\
\text { disagree }\end{array}$ & Total & Consensus \\
\hline $30 \%$ & $40 \%$ & $10 \%$ & $16 \%$ & $4 \%$ & $100 \%$ & $70 \%$ \\
\hline
\end{tabular}

Statement 52

After gastric resection, oral feeding should be started as soon as the patient has conditions and the intestinal transit is restored.

\begin{tabular}{|c|c|c|c|c|c|c|}
\hline Fully agree & $\begin{array}{c}\text { Partially } \\
\text { agree }\end{array}$ & Undecided & Disagree & $\begin{array}{l}\text { Strongly } \\
\text { disagree }\end{array}$ & Total & Consensus \\
\hline $48 \%$ & $35 \%$ & $2 \%$ & $6 \%$ & $9 \%$ & $100 \%$ & $83 \%$ \\
\hline
\end{tabular}

\section{Chemoradiotherapy statements}

\section{Statement 53}

Perioperative chemotherapy (before and after surgery) is indicated for stage $\geq \mathrm{IB}$ resectable tumors of the distal third.

\begin{tabular}{|c|c|c|c|c|c|c|}
\hline Fully agree & $\begin{array}{c}\text { Partially } \\
\text { agree }\end{array}$ & Undecided & Disagree & $\begin{array}{l}\text { Strongly } \\
\text { disagree }\end{array}$ & Total & Consensus \\
\hline $34 \%$ & $34 \%$ & $6 \%$ & $20 \%$ & $6 \%$ & $100 \%$ & $68 \%$ \\
\hline
\end{tabular}

\section{Statement 54}

Perioperative chemotherapy (before and after surgery) is indicated for stage $\geq$ I resectable tumors of the middle and proximal third.

\begin{tabular}{|c|c|c|c|c|c|c|}
\hline Fully agree & $\begin{array}{c}\text { Partially } \\
\text { agree }\end{array}$ & Undecided & Disagree & $\begin{array}{l}\text { Strongly } \\
\text { disagree }\end{array}$ & Total & Consensus \\
\hline $38 \%$ & $40 \%$ & $4 \%$ & $18 \%$ & $0 \%$ & $100 \%$ & $78 \%$ \\
\hline
\end{tabular}

\section{Statement 55}

Stage $\geq I B$ patients who underwentsurgery withoutperioperative chemotherapy (up front surgery) have an indication for adjuvant chemotherapy.

\begin{tabular}{|c|c|c|c|c|c|c|}
\hline Fully agree & $\begin{array}{c}\text { Partially } \\
\text { agree }\end{array}$ & Undecided & Disagree & $\begin{array}{l}\text { Strongly } \\
\text { disagree }\end{array}$ & Total & Consensus \\
\hline $44 \%$ & $36 \%$ & $6 \%$ & $10 \%$ & $4 \%$ & $100 \%$ & $80 \%$ \\
\hline
\end{tabular}


Statement 56

Adjuvant radiotherapy is recommended in cases with an indication for adjuvant chemotherapy and who did not have an adequate lymph node dissection during surgery.

\begin{tabular}{|c|c|c|c|c|c|c|}
\hline Fully agree & $\begin{array}{l}\text { Partially } \\
\text { agree }\end{array}$ & Undecided & Disagree & $\begin{array}{l}\text { Strongly } \\
\text { disaqree }\end{array}$ & Total & Consensus \\
\hline $38 \%$ & $44 \%$ & $8 \%$ & $10 \%$ & $0 \%$ & $100 \%$ & $82 \%$ \\
\hline
\end{tabular}

Statement 57

Patients with metastatic gastric cancer, in good clinical condition, have an indication for palliative chemotherapy.

\begin{tabular}{|c|c|c|c|c|c|c|}
\hline Fully agree & $\begin{array}{l}\text { Partially } \\
\text { agree }\end{array}$ & Undecided & Disagree & $\begin{array}{l}\text { Strongly } \\
\text { disagree }\end{array}$ & Total & Consensus \\
\hline $76 \%$ & $18 \%$ & $6 \%$ & $0 \%$ & $0 \%$ & $100 \%$ & $94 \%$ \\
\hline
\end{tabular}

\section{Statement 58}

Patients with metastatic gastric cancer who respond well to palliative chemotherapy and have little residual disease are candidates for conversion therapy with the aim of achieving an $\mathrm{RO}$ resection.

\begin{tabular}{|c|c|c|c|c|c|c|}
\hline Fully agree & $\begin{array}{l}\text { Partially } \\
\text { agree }\end{array}$ & Undecided & Disagree & $\begin{array}{l}\text { Strongly } \\
\text { disagree }\end{array}$ & Total & Consensus \\
\hline $48 \%$ & $36 \%$ & $6 \%$ & $8 \%$ & $2 \%$ & $100 \%$ & $84 \%$ \\
\hline
\end{tabular}

\section{Statement 59}

Hyperthermic intraperitoneal chemotherapy (HIPEC) should be used only in research protocols, as there is still no consistent evidence of its real benefit.

\begin{tabular}{|c|c|c|c|c|c|c|}
\hline Fully agree & $\begin{array}{c}\text { Partially } \\
\text { agree }\end{array}$ & Undecided & Disagree & $\begin{array}{l}\text { Strongly } \\
\text { disagree }\end{array}$ & Total & Consensus \\
\hline $74 \%$ & $12 \%$ & $6 \%$ & $6 \%$ & $2 \%$ & $100 \%$ & $86 \%$ \\
\hline
\end{tabular}

\section{Statement 60}

Metastatic gastric cancer patients HER-2 positive are indicated for target therapy treatment (monoclonal antibody) associated with palliative chemotherapy.

\begin{tabular}{|c|c|c|c|c|c|c|}
\hline Fully agree & $\begin{array}{c}\text { Partially } \\
\text { agree }\end{array}$ & Undecided & Disagree & $\begin{array}{l}\text { Strongly } \\
\text { disagree }\end{array}$ & Total & Consensus \\
\hline $72 \%$ & $20 \%$ & $8 \%$ & $0 \%$ & $0 \%$ & $100 \%$ & $92 \%$ \\
\hline
\end{tabular}

\section{Statement 61}

Immunotherapy for patients with metastatic gastric cancer should be used only in research protocols, as there is still no consistent evidence of its real benefit.

\begin{tabular}{|c|c|c|c|c|c|c|}
\hline Fully agree & $\begin{array}{l}\text { Partially } \\
\text { agree }\end{array}$ & Undecided & Disagree & $\begin{array}{l}\text { Strongly } \\
\text { disagree }\end{array}$ & Total & Consensus \\
\hline $42 \%$ & $28 \%$ & $8 \%$ & $16 \%$ & $6 \%$ & $100 \%$ & $70 \%$ \\
\hline
\end{tabular}

\section{Follow-up statements}

Statement 62

Patients with metastatic gastric cancer who have notresponded to palliative chemotherapy or in poor clinical conditions, should receive only palliative care with best support of care.

\begin{tabular}{|c|c|c|c|c|c|c|}
\hline Fully agree & $\begin{array}{c}\text { Partially } \\
\text { agree }\end{array}$ & Undecided & Disagree & $\begin{array}{l}\text { Strongly } \\
\text { disagree }\end{array}$ & Total & Consensus \\
\hline $84 \%$ & $12 \%$ & $2 \%$ & $2 \%$ & $0 \%$ & $100 \%$ & $96 \%$ \\
\hline
\end{tabular}

Statement 63

Patients undergoing radical surgery or after adjuvant therapy should not be followed due to the high cost and because there is no evidence that the follow-up improves survival.

\begin{tabular}{|c|c|c|c|c|c|c|}
\hline Fully agree & $\begin{array}{l}\text { Partially } \\
\text { agree }\end{array}$ & Undecided & Disagree & $\begin{array}{l}\text { Strongly } \\
\text { disagree }\end{array}$ & Total & Consensus \\
\hline $4 \%$ & $14 \%$ & $2 \%$ & $36 \%$ & $44 \%$ & $100 \%$ & $18 \%$ \\
\hline
\end{tabular}

Statement 64

Patients submitted to radical surgery can be followed through abdominal ultrasound, due to its accessibility and low cost.

\begin{tabular}{|c|c|c|c|c|c|c|}
\hline Fully agree & $\begin{array}{c}\text { Partially } \\
\text { agree }\end{array}$ & Undecided & Disagree & $\begin{array}{c}\text { Strongly } \\
\text { disagree }\end{array}$ & Total & Consensus \\
\hline $10 \%$ & $28 \%$ & $6 \%$ & $42 \%$ & $14 \%$ & $100 \%$ & $38 \%$ \\
\hline
\end{tabular}

\section{Statement 65}

In the postoperative period of patients submitted to radical surgery, the upper endoscopy is indicated when there is clinical suspicion of recurrence and digestive symptoms.

\begin{tabular}{|c|c|c|c|c|c|c|}
\hline Fully agree & $\begin{array}{c}\text { Partially } \\
\text { agree }\end{array}$ & Undecided & Disagree & $\begin{array}{c}\text { Strongly } \\
\text { disagree }\end{array}$ & Total & Consensus \\
\hline $48 \%$ & $30 \%$ & $0 \%$ & $20 \%$ & $2 \%$ & $100 \%$ & $78 \%$ \\
\hline
\end{tabular}

\section{Statement 66}

The long-term follow-up should be offered to patients undergoing radical surgery or after the end of adjuvant therapy for nutritional and psychological control and support, early detection of recurrence, treatment of complications and data collection.

\begin{tabular}{|c|c|c|c|c|c|c|}
\hline Fully agree & $\begin{array}{l}\text { Partially } \\
\text { agree }\end{array}$ & Undecided & Disagree & $\begin{array}{l}\text { Strongly } \\
\text { disagree }\end{array}$ & Total & Consensus \\
\hline $88 \%$ & $12 \%$ & $0 \%$ & $0 \%$ & $0 \%$ & $100 \%$ & $100 \%$ \\
\hline
\end{tabular}

\section{Statement 67}

The attempt of surgical resection in patients with single local recurrence and low surgical risk can be considered in selected cases.

\begin{tabular}{|c|c|c|c|c|c|c|}
\hline Fully agree & $\begin{array}{c}\text { Partially } \\
\text { agree }\end{array}$ & Undecided & Disagree & $\begin{array}{l}\text { Strongly } \\
\text { disagree }\end{array}$ & Total & Consensus \\
\hline $78 \%$ & $20 \%$ & $0 \%$ & $0 \%$ & $2 \%$ & $100 \%$ & $98 \%$ \\
\hline
\end{tabular}

\section{DISCUSSION}

The word consensus originates from the Latin (consensus) and, by definition, means the agreement or uniformity of opinions, thoughts, feelings and beliefs of the majority or all of the members of a collective. Consensus are quite common in medicine. They are usually performed by experts in certain areas and consist of creating diagnostic and treatments guidelines for certain diseases ${ }^{7}$.

Several international societies have published their respective consensus and guidelines ${ }^{2,3,4,8,12,13,19,20,22}$. In fact, it is paramount that each country has its own consensus. Although GC has similar characteristics between different races, there are peculiarities between different countries that must be addressed according to local conditions. These include the incidence of the disease, sanitary conditions, eating habits, cultural aspects, accessibility to diagnostic methods and treatment, among others. These differences can be seen in our country. Due to its enormous size, there is a huge difference between the aspects mentioned between the regions of Brazil. Therefore, it is essential that each location seeks to adapt the information contained in this consensus with its own reality, always seeking early diagnosis and the most effective treatment possible.

Unlike the I Consensus published in $2013^{21}$, in which the participants had the option to answer only yes or no to the questions, the authors of this consensus chose to provide more options for answers. This is because GC is a complex disease, with multiple factors that may influence its management. In short, often more than one path considered to be correct can be followed. In addition, this will allow, in future publications, each statement to be commented on based on evidence from the latest medical literature worldwide. 
CONCLUSION

The treatment of gastric cancer has evolved considerably in recent years. This consensus gathers the answers of the questionnaire prepared by ABCG of several specialists in gastric cancer treatment in Brazil. This is the commitment of the Brazilian Gastric Cancer Association: to disseminate knowledge and allow professionals to achieve better outcomes in terms of patients' survival and quality of life.

\section{ACKNOWLEDGMENT}

The authors would like to thank all consensus member for scientific support and Cynthia Chiaradia for the data collection and statistical support.

\section{Consensus Members (in alphabetical order)}

\begin{tabular}{|c|c|}
\hline $\begin{array}{l}\text { ÁlvaroAntônioBandeira } \\
\text { Ferraz }\end{array}$ & $\begin{array}{l}\text { Hospital das Clínicas, Universidade Federal de Pernambuco, } \\
\text { Recife, PE, Brasil }\end{array}$ \\
\hline Amir Zeide Charruf & $\begin{array}{l}\text { Hospital das Clínicas, Faculdade de Medicina, Universidade } \\
\text { de São Paulo, São Paulo, SP, Brasil }\end{array}$ \\
\hline André Brandalise & Hospital Centro Médico de Campinas, Campinas, SP, Brasil \\
\hline André Maciel da Silva & $\begin{array}{l}\text { Instituto Nacional do Câncer-INCA, Hospital Federal do } \\
\text { Andaraí, Rio de Janeiro, RJ, Brasil }\end{array}$ \\
\hline Barlon Alves & Hospital Regional de Ceilândia, Brasília, DF, Brasil \\
\hline $\begin{array}{l}\text { CarlosAugustoMartinez } \\
\text { Marins }\end{array}$ & $\begin{array}{l}\text { Hospital Federal dos Servidores do Estado/ RJ, Instituto } \\
\text { Nacional do Câncer- INCA, Rio de Janeiro, RJ, Brasil }\end{array}$ \\
\hline Celso Vieira Leite & Universidade Estadual de São Paulo, Botucatu, SP, Brasil \\
\hline $\begin{array}{l}\text { Claudio José Caldas } \\
\text { Bresciani }\end{array}$ & $\begin{array}{l}\text { Hospital das Clínicas, Faculdade de Medicina, Universidade } \\
\text { de São Paulo, São Paulo, SP, Brasil }\end{array}$ \\
\hline Daniel Szor & $\begin{array}{l}\text { Hospital das Clínicas, Faculdade de Medicina, Universidade } \\
\text { de São Paulo, São Paulo, SP, Brasil }\end{array}$ \\
\hline $\begin{array}{l}\text { Donato Roberto } \\
\text { Mucerino }\end{array}$ & $\begin{array}{l}\text { Hospital das Clínicas, Faculdade de Medicina, Universidade } \\
\text { de São Paulo, São Paulo, SP, Brasil }\end{array}$ \\
\hline Durval R. Wohnrath & $\begin{array}{l}\text { Fundação Pio XII, Hospital de Câncer de Barretos, Barretos, } \\
\text { SP, Brasil }\end{array}$ \\
\hline Elias Jirjoss Ilias & $\begin{array}{l}\text { Departamento de Cirurgia, Santa Casa de São Paulo, São } \\
\text { Paulo, SP, Brasil }\end{array}$ \\
\hline $\begin{array}{l}\text { Euclides Dias Martins } \\
\text { Filho }\end{array}$ & Universidade Federal de Pernambuco, Recife, PE, Brasil \\
\hline Fabio Pinatel Lopasso & $\begin{array}{l}\text { Hospital das Clínicas, Faculdade de Medicina, Universidade } \\
\text { de São Paulo, São Paulo, SP, Brasil }\end{array}$ \\
\hline $\begin{array}{l}\text { Felipe José Fernandez } \\
\text { Coimbra }\end{array}$ & AC Camargo Cancer Center, São Paulo, SP, Brasil \\
\hline FernandoE.CruzFelippe & $\begin{array}{l}\text { Departamento de Cirurgia Oncológica Digestiva Alta do } \\
\text { Hospital de Amor de Barretos, Barretos, SP, Brasil }\end{array}$ \\
\hline $\begin{array}{l}\text { Flávio Daniel Saavedra } \\
\text { Tomasisch }\end{array}$ & $\begin{array}{l}\text { Universidade Federal do Paraná, Serv. Oncologia Digestiva } \\
\text { do Hospital Erasto Gaertner, Curitiba, R. Brasil }\end{array}$ \\
\hline Flavio Roberto Takeda & $\begin{array}{l}\text { Hospital das Clínicas, Faculdade de Medicina, Universidade } \\
\text { de São Paulo, São Paulo, SP, Brasil }\end{array}$ \\
\hline Geraldo Ishak & Universidade Federal do Pará, Belém, PA, Brasil \\
\hline $\begin{array}{l}\text { Gustavo Andreazza } \\
\text { Laporte }\end{array}$ & $\begin{array}{l}\text { Departamento de Cirurgia, Santa Casa de Porto Alegre, } \\
\text { Porto Alegre, RS, Brasil }\end{array}$ \\
\hline HerbethJoséToledoSilva & Universidade Estadual de Alagoas, Maceió, AL, Brasil \\
\hline Ivan Cecconello & $\begin{array}{l}\text { Hospital das Clínicas, Faculdade de Medicina, Universidade } \\
\text { de São Paulo, São Paulo, SP, Brasil }\end{array}$ \\
\hline $\begin{array}{l}\text { Joaquim José Gama } \\
\text { Rodrigues }\end{array}$ & $\begin{array}{l}\text { Hospital das Clínicas, Faculdade de Medicina, Universidade } \\
\text { de São Paulo, São Paulo, SP, Brasil }\end{array}$ \\
\hline JoséCarlos Del Grande & Universidade Federal de São Paulo, São Paulo, SP, Brasil \\
\hline $\begin{array}{l}\text { Leonardo Milhomem } \\
\text { da Motta }\end{array}$ & Universidade Federal de Goiás, Goiânia, GO, Brasil \\
\hline Leonardo Rocha Ferraz & Hospital Federal de Bonsucesso, Rio de Janeiro, RJ, Brasil \\
\hline Luis Fernando Moreira & $\begin{array}{l}\text { Universidade Federal do Rio Grande do Sul, Porto Alegre, } \\
\text { RS, Brasil }\end{array}$ \\
\hline Luis Roberto Lopes & Universidade Estadual de Campinas, Campinas, SP, Brasil \\
\hline Marcelo Garcia Toneto & $\begin{array}{l}\text { Hospital São Lucas da PUC-RS, Faculdade de Medicina da } \\
\text { PUC-RS, Porto Alegre, RS, Brasil }\end{array}$ \\
\hline Marcelo Mester & $\begin{array}{l}\text { Hospital das Clínicas, Faculdade de Medicina, Universidade } \\
\text { de São Paulo, São Paulo, SP, Brasil }\end{array}$ \\
\hline $\begin{array}{l}\text { Marco Antônio } \\
\text { Gonçalves Rodrigues }\end{array}$ & $\begin{array}{l}\text { Universidade Federal de Minas Gerais, Belo Horizonte, } \\
\text { MG, Brasil }\end{array}$ \\
\hline
\end{tabular}

Marineide Prudêncio Departamento de Cirurgia, Santa Casa de São Paulo, São de Carvalho

Paulo, SP, Bra

MauriceYoussefFranciss Departamento de Cirurgia, Santa Casa de São Paulo, São Paulo, SP, Brasil

NoraManoukianForones Universidade Federal de São Paulo, São Paulo, SP, Brasil Oly Campos Corletta Universidade Federal de Rio Grande do Sul, Porto Alegre, Oly Campos Corletta RS, Brasil

Osmar Kenji Yagi Hospital das Clínicas, Faculdade de Medicina, Universidade de São Paulo, São Paulo, SP, Brasil

Osvaldo Antonio Prado Departamento de Cirurgia, Santa Casa de São Paulo, São Castro Paulo, SP, Brasil

Osvaldo Malafaia Faculdade Evangélica Mackenzie do Paraná, Curitiba, PR, Brasil

Paulo Pimentel Assumpção

Paulo RobertoSavassi- Universidade Federal de Minas Gerais, Belo Horizonte, Rocha MG, Brasil

Ramiro Colleoni Neto Universidade Federal de São Paulo, São Paulo, SP, Brasil

Rodrigo JosedeOliveira Hospital das Clínicas, Faculdade de Medicina, Universidade de São Paulo, São Paulo, SP, Brasil

Rubens Antonio Aissar Hospital das Clínicas, Faculdade de Medicina, Universidade Sallun de São Paulo, São Paulo, SP, Brasi

Rui Weschenfelder Hospital Moinhos de Vento, Porto Alegre, RS, Brasil

Saint Clair Vieira de UniversidadeFederal deSanta Catarina, Florianópolis, SC, Brasil Oliveira

ThiagoBoechatdeAbreu Hospital Federal de Bonsucesso, Rio de Janeiro, RJ, Brasil

Tiago Biachide Castria Hospital das Clínicas, Faculdade de Medicina, Universidade Tiago Biachi de Castria de São Paulo, São Paulo, SP, Brasil

Ulysses Ribeiro Junior Hospital das Clínicas, Faculdade de Medicina, Universidade de São Paulo, São Paulo, SP, Brasil

Williams Barra Universidade Federal do Pará, Belem, PA, Brasil

Wilson Luiz da Costa Department of Medicine, Baylor College of Medicine, únior Houston, Texas, USA

Wilson Rodrigues de Departamento de Cirurgia, Santa Casa de São Paulo, São Freitas Júnior Paulo, SP, Brasil

\section{REFERENCES}

1. Amorim CA, Moreira JP, Rial L, et al. Ecological study of gastric cancer in Brasil: geographic and time trend analysis. World J Gastroenterol. 2014;20(17):5036-5044.

2. Ajani JA, D'Amico TA, Almhanna K, Bentrem DJ, Chao J, Das P, etal. Gastric Cancer, Version 3.2016, NCCN Clinical Practice Guidelines in Oncology. J Natl Compr Canc Netw. 2016;14(10):1286-312.

3. Association JGC. Japanese gastric cancer treatment guidelines 2018 (5th edition). Gastric Cancer. 2020

4. Baiocchi GL, D'Ugo D, Coit D, Hardwick R, Kassab P, Nashimoto A, et al. Follow-up after gastrectomy for cancer: the Charter Scaligero Consensus Conference. Gastric Cancer. 2016;19(1):15-20.

5. Barchi LC, Gama-Rodrigues J, Carvalho FA, Barchi MC, Oliveira OC, Carneiro MF. Cystic gastric stromal tumor negative C-Kit. Arq Bras Cir Dig. 2012;25(4):300-302

6. BrayF, FerlayJ,Soerjomataram I,SiegelRL, Torre LA,JemalA.Global cancer statistics2018:GLOBOCANestimatesofincidenceandmortalityworldwide for 36 cancers in 185 countries. CA Cancer J Clin. 2018;68(6):394-424.

7. Council ofEurope, Developing a methodology fordrawing up guidelines on bestmedical practice (Recommendation Rec(2001)13andexplanatory memorandum), Strasbourg: Council of Europe Publishing, 2002.

8. De Manzoni G, Marrelli D, Baiocchi GL, Morgagni P, Saragoni L, Degiuli M, et al. The Italian Research Group for Gastric Cancer (GIRCG) guidelines for gastriccancerstagingand treatment:2015.GastricCancer.2017;20(1):20-30.

9. Dias AR, Azevedo BC, Alban LBV, et al. Gastric neuroendocrine tumor review and update. Arq Bras Cir Dig. 2017;30(2):150-154.

10. FIGUEROA-GIRALT, Manuel et al. Introduction of the new lymphoparietal index for gastric cancer patients. ABCD, arq. bras. cir. dig., 2019, vol.32, no.2. ISSN 0102-6720.

11. Instituto Nacional de Câncer José Alencar Gomes da Silva (INCA). Estimativa2018:incidência decâncernoBrasil. Rio de Janeiro(RJ),2018.In.

12. Kulig J, Wallner G, Drews M, Frączek M, Jeziorski A, Kielan W, et al. Polish Consensus on Treatment of Gastric Cancer; update 2017. Pol Przegl Chir. 2017;89(5):59-73.

13. Lee JH, Kim JG, Jung HK, Kim JH, Jeong WK, Jeon TJ, et al. Clinical practice guidelines for gastric cancer in Korea: an evidence-based approach. J Gastric Cancer. 2014;14(2):87-104.

14. Moore MA, Eser $\mathrm{S}$, Igisinov $\mathrm{N}$, et al. Cancer epidemiology and contro in North-Western and Central Asia - past, present and future. Asian Pac J Cancer Prev. 2010;11 Suppl 2:17-32. 
15. Norero E, Quezada JL, Cerda J, et al. Risk factors for severe postoperative complicationsaftergastrectomyforgastricandesophagogastricjunction cancers. Arq Bras Cir Dig. 2019;32(4):e1473. Published 2019 Dec 20. doi:10.1590/0102-672020190001e1473.

16. Peduk S, Dincer M, Tatar $C$, et al. The role of serum CK-18, MMP-9 and TIPM-1 levelsin predicting rO resection in patients with gastric cancer.Arq Bras Cir Dig. 2018;31(4):e1401. Published 2018 Dec 6. doi:10.1590/0102$672020180001 \mathrm{e} 1401$.

17. Ramos $M$, Pereira MA, Charruf $A Z$, et al. Conversion therapy for gastric cancer: expanding the treatment possibilities. ABCD. 2019;32(2):e1435

18. Sano T, Coit DG, Kim HH, etal. Proposal of a new stage grouping of gastric cancer for TNM classification: International Gastric Cancer Association staging project. Gastric Cancer. 2017;20(2):217-225.
19. Smyth EC, Verheij M, Allum W, Cunningham D, Cervantes A, Arnold D, et al. Gastric cancer: ESMO Clinical Practice Guidelines for diagnosis, treatment and follow-up. Ann Oncol. 2016;27(suppl 5):v38-v49.

20. Zaanan A, Bouché O, Benhaim L, Buecher B, Chapelle N, Dubreuil O, et al. Gastriccancer:French intergroup clinical practiceguidelinesfordiagnosis, treatments and follow-up (SNFGE, FFCD, GERCOR, UNICANCER, SFCD, SFED, SFRO). Dig Liver Dis. 2018;50(8):768-79.

21. Zilberstein B, Malheiros C, Lourenço LG, et al. Brasilian consensus in gastric cancer: guidelines for gastric cancer in Brasil. Arq Bras Cir Dig. 2013;26(1):2-6

22. Wang FH, Shen L, Li J, Zhou ZW, Liang H, Zhang XT, et al. The Chinese Society of Clinical Oncology (CSCO): clinical guidelines for the diagnosis and treatment of gastric cancer. Cancer Commun (Lond). 2019;39(1):10. 